\title{
Prediction of cutting forces in ball-end milling by means of geometric analysis
}

\author{
Chung-Liang Tsai ${ }^{a, *}$, Yunn-Shiuan Liao ${ }^{b}$ \\ a Department of Mechanical Engineering, Lee-Ming Institute of Technology, No. 2-2, Lijhuan Road, Taishan, Taipei County 243, \\ Taiwan, ROC \\ b Department of Mechanical Engineering, National Taiwan University, No. 1, Sec. 4, Roosevelt Road, Taipei City 106, Taiwan, ROC
}

\section{A R T I C L E I N F O}

Article history:

Received 19 February 2007

Received in revised form

1 September 2007

Accepted 11 November 2007

Keywords:

Ball-end milling

Cutting force

Minimum energy method

\begin{abstract}
A B S T R A C T
A new geometric model of ball-end milling is proposed. The relationships among undeformed chip thickness, rake angle, cutting velocity, shear plane area and chip flow angle in ball-end milling are clearly described. The shear plane area of the shear deformation zone and the effective frictional area on tool face are calculated. The three-dimensional cutting forces in the tool axis system are then obtained by minimum energy method. The transformation matrices, including the effects of the axial depth of cut, helix angle, tool rotational angle, and indentation action of the tool tip, are presented. The three-dimensional cutting forces can be predicted via matrix transformation. A force model for a two-flute cutter is developed and a four-flute model is also obtained by superposing the two-flute model. Based on the basic geometric modeling, the cutting forces along horizontal and vertical directions are predicted. Several unveiled phenomena, such as the influence on cutting forces of the different engaged regions between cutting edge and undeformed chip thickness, and indentation action on tool tip that influences the Z-direction force, have been illustrated. Experiments are conducted to verify the developed model. It shows that the predicted cutting forces agree with the experimental data both in trends and values.
\end{abstract}

(c) 2007 Elsevier B.V. All rights reserved.

\section{Introduction}

Ball-end milling process is widely used in machining parts of sculptured surfaces. Much work has been done in the past related to the cutting forces of the ball-end milling process. Yang and Park (1991) divided the screw cutting edge of a ball-end milling cutter into a series of infinitesimal elements and assumed that each cutting edge was straight. The oblique cutting process in this small element was analyzed as an orthogonal cutting process. Lee and Altintas (1996) introduced an oblique concept to ball-end milling process. The flute was expressed in a parametric form and divided into small oblique cutting edges. Then the mathematical relationships were developed to relate the geometry of each oblique element to variables obtained from orthogonal cutting tests. Engin and Altintas (2001) presented a generalized mathematical model of helical end mills. Kivanc and Budak (2004) developed some methods of modeling structural properties of milling tools. The geometric analysis was carried out by finite element analysis. Fontaine et al. (2006) presented a predictive force model in which the tool geometry was decomposed into a series of axial elementary cutting edges. The chip formation was obtained from an oblique cutting process characterized by local undeformed chip section and local angles. All the previous researches have a characteristic in common. That is, the ball-end cutting model is assumed to be composed of a

\footnotetext{
* Corresponding author. Tel.: +886 2 29097811x2200; fax: +886 222966301.

E-mail address: cltsai88@ms2.kntech.com.tw (C.-L. Tsai). 
series of small cutting edges, and the orthogonal or oblique cutting process in the small element is analyzed. Summing up the cutting force in every element, the total cutting force of ball-end cutting edge can be evaluated. Since the cutting edge is divided into infinitesimal straight cutting unit, the engaged region between the cutter edge and undeformed chip thickness cannot be understood, and also indentation action on tool tip during cutting process was not clearly described. In reality, the different engaged region may cause difference in amplitude and shape of the cutting force. In addition, indentation action on tool tip will specially influence the Z-direction force, which cannot be exactly predicted by Yang and Park's model (Yang and Park, 1991) and they attributed the departure between prediction and experiment in Z-direction force to an action of "a cutting singularity". Hence, the purpose of this paper is to develop a geometric force model in ball-end milling to reveal several unveiled phenomena of cutting forces in horizontal and vertical feed cuttings, such as the influence of the different engaged region (It is defined as a center-leading angle $\psi$ in this paper) and indentation action on tool tip that influences the Z-direction force.

Usui et al. (1978) and Usui and Hirota (1978) proposed a geometric model of chip forming process in three-dimensional cutting with a single point tool. Based on this model, an energy method similar to the upper bound approach in metal forming is developed to predict three axes cutting forces. This paper extends Usui's geometric model to develop a new three-dimensional cutting model in ball-end milling process. Different from the previous works that the flute was divided into small cutting edges, a complete cutting flute is considered. An average cutting velocity is assumed to represent the cutting velocity. Although there are some departures between the measurements and predictions with this assumption, this greatly simplifies the derivation of force model with only little effect on the purpose of explaining the cutting phenomena as will be shown in the paper.

\section{The shear plane area and the effective friction area}

When the workpiece is cut, it is torn apart by the sharp cutting edge. The cutting energy per unit time $U$ can be divided mainly into two parts; the shear energy per unit time, $U_{\mathrm{s}}$, and the friction energy per unit time, $U_{f}$, i.e.

$U=U_{\mathrm{s}}+U_{\mathrm{f}}$

Denoting the shear velocity and the shear force as $V_{s}$ and $F_{\mathrm{s}}$, and the chip velocity and the friction force as $V_{c}$ and $F_{c}$, respectively, then the shear energy per unit time in the deformation zone and the friction energy per unit time on the tool face can be expressed as

$U_{\mathrm{s}}=F_{\mathrm{s}} V_{\mathrm{S}}$

$U_{\mathrm{f}}=F_{\mathrm{c}} \mathrm{V}_{\mathrm{c}}$

To calculate $U_{\mathrm{s}}$ and $U_{\mathrm{f}}$, shear force and friction force must be both obtained beforehand. But owing to the fact that it

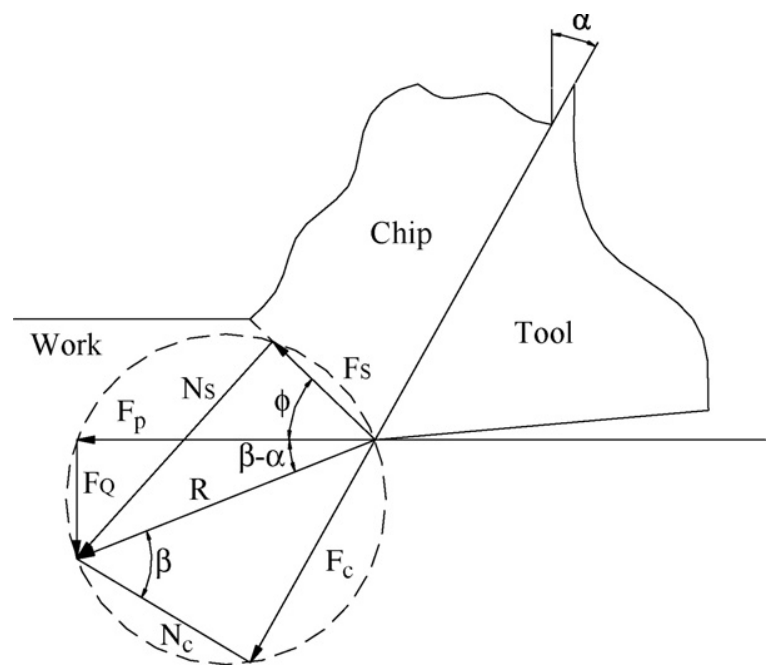

Fig. 1 - Composite cutting force circle (Shaw, 1984).

cannot be achieved by direct measurements, the geometric relationships between tool and chip described in this threedimensional cutting force model are used to find the shear force and friction force instead.

To simplify the cutting behaviors occurring in the threedimensional cutting process, the two-dimensional orthogonal cutting force circle of Shaw (1984), shown in Fig. 1 is discussed first.

Let the area of shear plane and the shear stress along this plane be donated by $A_{\mathrm{S}}$ and $\tau_{\mathrm{S}}$ respectively, then the shear force $F_{\mathrm{S}}$ can be expressed as

$F_{\mathrm{S}}=\tau_{\mathrm{S}} A_{\mathrm{S}}$

Referring to Fig. 1, the shear force $F_{\mathrm{S}}$ is the component of the resultant force $R$ along shear plane. That is,

$F_{\mathrm{S}}=R \cos (\phi+\beta-\alpha)$

where $\phi$ is the shear angle, $\beta$ the friction angle and $\alpha$ is the rake angle.Substituting Eq. (5) into Eq. (4), the resultant force $R$ can be written as

$R=\frac{\tau_{\mathrm{S}} A_{\mathrm{S}}}{\cos (\phi+\beta-\alpha)}$

Similarly, the friction force is

$F_{\mathrm{C}}=R \sin \beta$

Substituting Eq. (6) into (7), yields

$F_{\mathrm{C}}=\tau_{\mathrm{S}} A_{\mathrm{S}}\left[\frac{\sin \beta}{\cos (\phi+\beta-\alpha)}\right]$

Assuming

$\mathrm{Q}_{\mathrm{p}}=\mathrm{A}_{\mathrm{s}}\left[\frac{\sin \beta}{\cos (\phi+\beta-\alpha)}\right]$ 
Then the friction force $F_{\mathrm{c}}$ can be expressed as

$F_{\mathrm{c}}=\tau_{\mathrm{s}} \mathrm{Q}_{\mathrm{p}}$

In the above equation, $Q_{p}$ is the component of shear plane area along frictional direction and is defined as an effective friction area.

The shear velocity $V_{s}$ and the chip velocity $V_{c}$ are related to cutting velocity $\mathrm{V}$, rake angle $\alpha$ and shear angle $\phi$, by the followings:

$\mathrm{V}_{\mathrm{S}}=\frac{\mathrm{V} \cos \alpha}{\cos (\phi-\alpha)}$

$\mathrm{V}_{\mathrm{c}}=\frac{\mathrm{V} \sin \phi}{\cos (\phi-\alpha)}$

Substituting Eq. (4) and (11) into Eq. (2), and Eq. (10) and (12) into Eq. (3) yields

$U_{\mathrm{s}}=\frac{\tau_{\mathrm{s}} \mathrm{A}_{\mathrm{S}} \mathrm{V} \cos \alpha}{\cos (\phi-\alpha)}$

and

$U_{\mathrm{f}}=\tau_{\mathrm{s}} \mathrm{Q}_{\mathrm{p}} \mathrm{V}\left[\frac{\sin \phi}{\cos (\phi-\alpha)}\right]$

From Eq. (13) and (14), it is obvious that $U_{\mathrm{s}}$ is a function of shear area $A_{s}$, while $U_{f}$ is a function of effective friction area $Q_{p}$. To obtain $A_{s}$ and $Q_{p}$ in more complicated three-dimensional cutting model, it is necessary to construct a three-dimensional geometric model that relates geometry angles and cutting conditions with shear area and effective friction area. It is also noted that, in two-dimensional cutting model, the cutting velocity $\mathrm{V}$ in equations (13) and (14) is constant. But in three-dimensional model, the velocity along cutting edge of ball-end cutter is varying. Without losing generality and the trends of cutting forces, $V$ is assumed to be the average cutting velocity of cutting edge to simply the derivation of the three-dimensional force model.

\section{Three-dimensional geometric model}

Based on Usui's concept, a three-dimensional geometric cutting model for ball-end milling is constructed. Fig. 2 shows a three-dimensional cutting geometric diagram feeding in horizontal direction. The chip is produced by shearing process in the shear plane (a curly bow area "abc") of the curly screw cutting edge with a horizontal feed. The cutting velocity $\mathrm{V}$ is tangent to the contact point of undeformed chip and ball-end cutting edge. The chip flows out of the tool face with a chip flow velocity $\mathrm{V}_{\mathrm{c}}$ and an angle $\eta_{\mathrm{c}}$ with respect to a "bu" line in the tool face "tbu". The "bu" line is parallel to the horizontal axis "ON". In the plane "kbl", the angle between the direction parallel to shear plane and the tangent line from the contact point $b$ (the direction of velocity $V$ ) is defined as the effective shear angle $\phi_{\mathrm{e}}$. In the plane "wbl" constituted by $\mathrm{V}$ and $\mathrm{V}_{\mathrm{c}}$, the effective rake angle $\alpha_{\mathrm{e}}$ ("kbw" angle in the figure) can be defined as an angle from tool face to the "bk" line

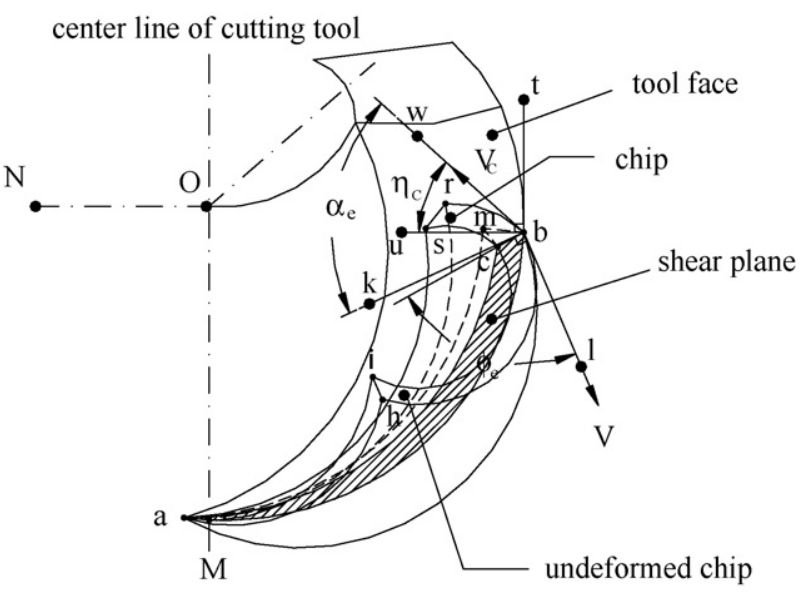

Fig. 2 - A three-dimensional cutting geometric diagram of ball-end milling cutting tool feeding in horizontal direction.

direction which is vertical to the direction of cutting velocity V. In this cutting process, the undeformed chip thickness (cross section "aih") is cut by the screw cutting edge (curly line "ab"), producing an effective frictional zone (area "abm") on the tool face and piling up a curly chip (cross section "ars"). It is noted that the cutting edge is extended from the point "a" to the highest point "b", but not from center point " $\mathrm{M}$ " which is intersected by center line of cutter and periphery of cutting edge. The angle "aOM" for useful chip releasing, making a lead over center line a certain angle, is defined as "chip release wall angle". A geometric diagram of ball-end milling cutting tool cutting in vertical direction is shown in Fig. 3. It is worthy of noting the difference between the model in Figs. 2 and 3. The cutting edge in Fig. 3 advances a distance of feed from the former edge (dash line "pnm") to the next cutting edge (curly line "ab"). Applying the same interpretation as in Fig. 2, we may find a special phenomenon that the material will be squeezed by the side of chip release wall (curly line "ae") in the vertical feeding direction. The undeformed chip thickness and the chip are squeezed simultaneously so that a squeezing line "ndj" is formed. The line is divided into two parts; one is squeezed while the other is not.

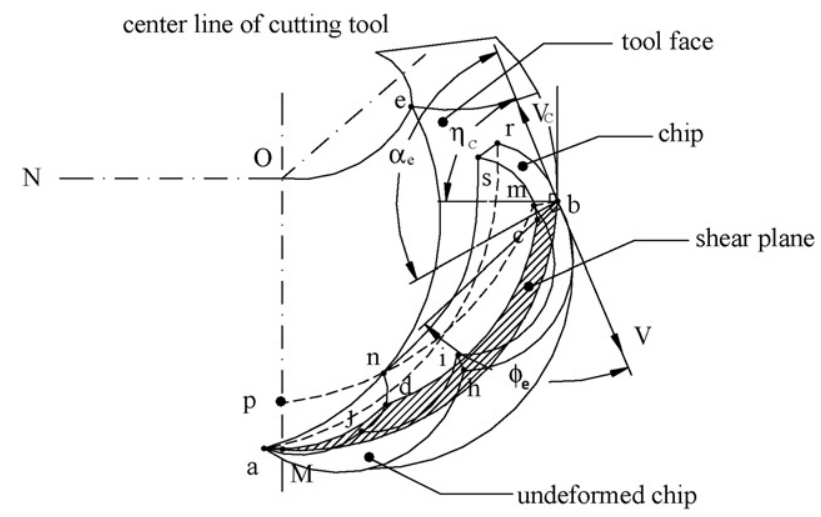

Fig. 3 - A three-dimensional cutting geometric diagram of ball-end milling cutting tool feeding in vertical direction. 


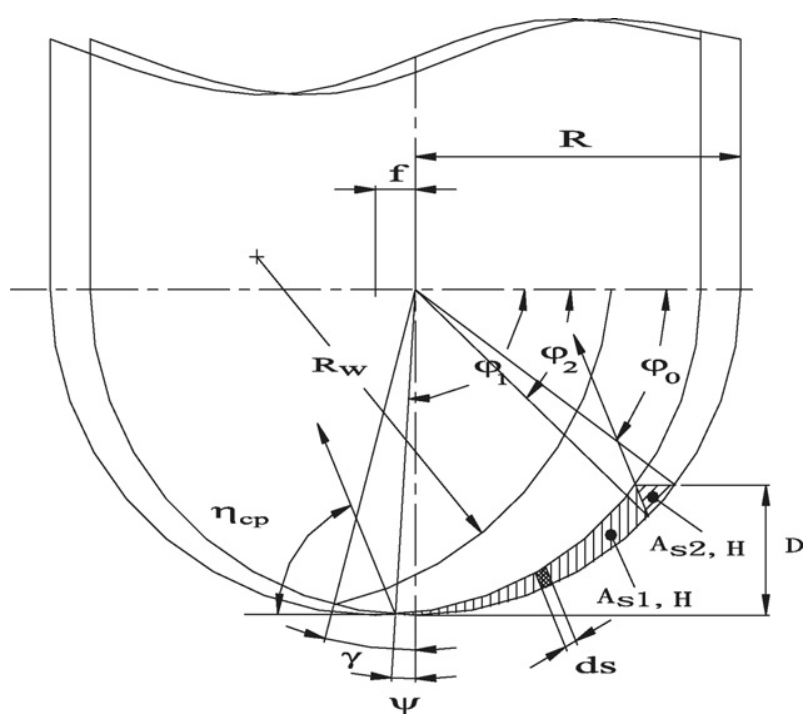

Fig. 4 - The projected model of shear plane feeding in horizontal direction.

\section{The projected model of shear plane}

\subsection{Feeding in the horizontal direction}

Using the geometric angles and tool cutting angles described in the model of Fig. 2, a projected model of shear plane is constructed in Fig. 4. In a cutting condition of having a horizontal feed $f$ and axial depth of cut $D$ with a ball-end cutter of radius $R$, it shows that a plane are projected from the tool face of the horizontal cutting model to a vertical plane "NOM" in Fig. 2. Because of horizontal direction cutting, the range of cutting area exceeds center line of the cutter, and an angle " $\psi$ " named as "center-lead angle" is produced. The "chip release wall angle" (i.e. the angle of "aOM" shown in Fig. 2) is represented by the notation of " $\gamma$ " in Fig. 4. Because the angle " $\gamma$ " is

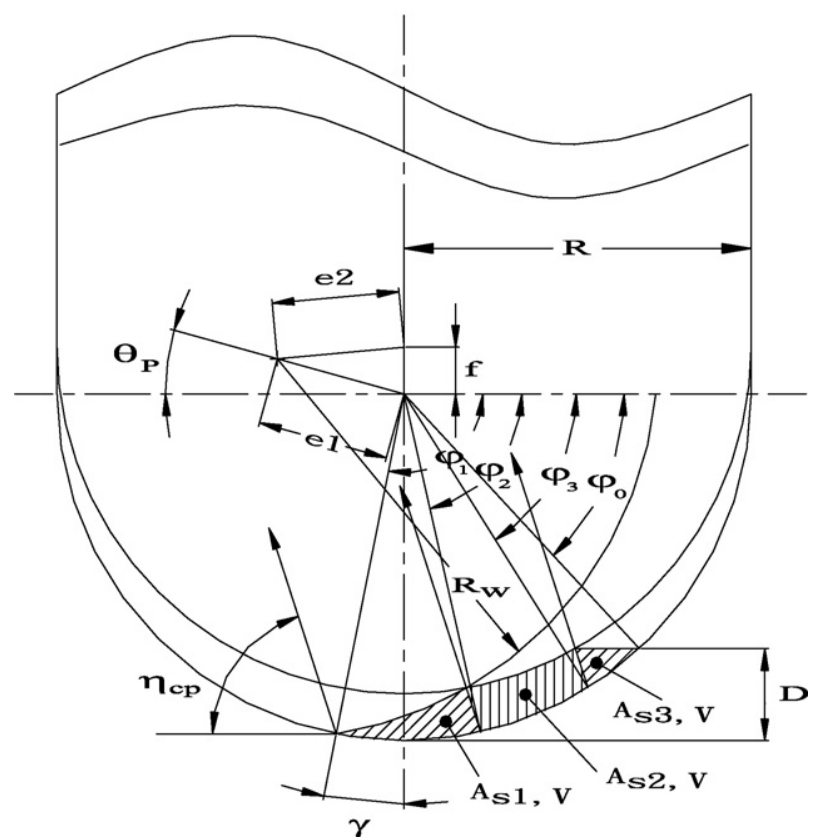

Fig. 5 - The projected model of shear plane feeding in vertical direction.

where in the above expression

$$
\begin{aligned}
\mathrm{ds}= & R\left\{\cos ^{2}\left(\eta_{\mathrm{CP}}-\varphi\right)+\left[\cos \phi_{\mathrm{e}} \sin \left(\eta_{\mathrm{CP}}-\varphi\right)\right.\right. \\
& \left.\left.+\sin \phi_{\mathrm{e}}\left(\sin i \cos \varphi-\tan \alpha_{\mathrm{r}}\left(\sin ^{2} i+1\right) \sin \varphi\right) / \cos i\right]^{2}\right\}^{1 / 2} \mathrm{~d} \varphi
\end{aligned}
$$

Details of functions of $f_{1}(\varphi)$ and $f_{2}(\varphi)$ are given in Appendix.

The effective frictional area $Q_{p}$ on the tool face can be written as:

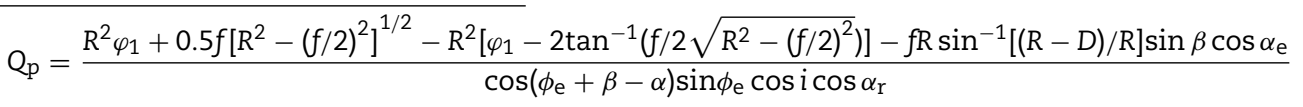

bigger than center-lead angle " $\psi$ ", the chip produced by cutting edge will not be squeezed by chip release wall. The shear plane shown in section line of Fig. 4 is divided into two parts, sections $A_{\mathrm{S} 1, \mathrm{H}}$ and $A_{\mathrm{s} 2, \mathrm{H}}$, according to the chip flow direction $\eta_{\mathrm{CP}}$ on the projected plane. We may have the relation between $\eta_{\mathrm{C}}$ and $\eta_{\mathrm{CP}}$ on the tool face, that is,

$\eta_{\mathrm{CP}}=\tan ^{-1}\left[\frac{\left(\tan \eta_{\mathrm{c}}-\sin \alpha_{\mathrm{r}} \tan i\right) \cos i}{\sin \alpha_{\mathrm{r}}}\right]$

where $\alpha_{\mathrm{r}}$ is the radial rake angle and $i$ is the inclination angle.

By using the notation of Fig. 4 , the area of shear plane $A_{S}$ may be obtained by the following equation:

$A_{s}=A_{s 1, H}+A_{s 2, H}=\int_{\varphi_{2}}^{\varphi_{1}} f_{1}(\varphi) d s+\int_{\varphi_{0}}^{\varphi_{2}} f_{2}(\varphi) d s$

\subsection{Feeding in vertical direction}

The three-dimensional cutting model of ball-end milling cutting in vertical direction also can be demonstrated by a geometric diagram of Fig. 3 and the projected model of shear plane is shown in Fig. 5. A notable phenomenon during vertical cutting in ball-end milling is the squeezing effect existing upon the side of chip release wall. The radius of chip release wall (denoted by " $R_{W}$ "), the distance between the center of cutter circle at the present feed and the center of chip release wall (denoted by " $e_{1}$ "), and the angle between $e_{1}$ and horizontal line (denoted by “ $\theta_{\mathrm{p}}$ "), must be considered. The distance between the center of cutter circle at the former feed and the center of chip release wall, represented by " $e_{2}$ ", can be obtained by the following relationship:

$e_{2}=\left(f^{2}+e_{1}^{2}-2 e_{1} f \sin ^{2} \theta_{p}\right)^{1 / 2}$ 
Using the notations in Fig. 5 with the same formuladeriving method in Section 4.1, the area of shear plane $A_{s}$ can be obtained by the following equation:

$A_{\mathrm{s}}=A_{\mathrm{s} 1, \mathrm{v}}+A_{\mathrm{s} 2, \mathrm{v}}+A_{\mathrm{s} 3, \mathrm{v}}=\int_{\varphi_{2}}^{\varphi_{1}} g_{1}(\varphi) \mathrm{d} s+\int_{\varphi_{3}}^{\varphi_{2}} g_{2}(\varphi)+\int_{\varphi_{0}}^{\varphi_{3}} g_{3}(\varphi) \mathrm{ds}(20)$

Functions of $g_{1}(\varphi), g_{2}(\varphi)$ and $g_{3}(\varphi)$ are again given in Appendix.The effective frictional area $Q_{p}$ can also be derived similarly, and it is,

$\mathrm{Q}_{\mathrm{p}}=\frac{\left(\mathrm{Q}_{1}+\mathrm{Q}_{2}+\mathrm{Q}_{3}-\mathrm{Q}_{4}-\mathrm{Q}_{5}-\mathrm{Q}_{6}\right) \sin \beta \cos \alpha_{\mathrm{e}}}{\cos \left(\phi_{\mathrm{e}}+\beta-\alpha_{\mathrm{e}}\right) \sin \phi_{\mathrm{e}} \cos i \cos \alpha_{\mathrm{r}}}$

where

$$
\begin{aligned}
& \mathrm{Q}_{1}=0.5 R^{2}\left(\varphi_{1}-\varphi_{0}\right), \\
& \mathrm{Q}_{2}=0.5 R f\left\{\frac{1-\left[f^{2}+R^{2}-\left(R \sin \theta_{\mathrm{E}} / \sin \gamma\right)^{2}\right]}{2 R f}\right\}^{1 / 2}, \\
& \mathrm{Q}_{3}=0.5 R f\left\{\frac{1-\left[f^{2}+R^{2}-\left(R \sin \theta_{\mathrm{D}} / \cos \varphi_{0}\right)^{2}\right]}{2 R f}\right\}^{1 / 2}, \\
& \mathrm{Q}_{4}=0.5 R^{2}\left[\frac{\pi}{2}-\varphi_{0}-\sin ^{-1}\left(\frac{f}{R \cos \varphi_{0}}\right)+\gamma-\sin ^{-1}\left(\frac{f}{R \sin \gamma}\right)\right], \\
& \mathrm{Q}_{5}=0.5 a_{1} b_{1}\left[1-\left(a_{1}^{2}+b_{1}^{2}-c_{1}^{2}\right) /\left(2 a_{1} b_{1}\right)\right]^{1 / 2} \text { and } \\
& \mathrm{Q}_{6}=0.5 a_{2} b_{2}\left[1-\left(a_{2}^{2}+b_{2}^{2}-c_{2}^{2}\right) /\left(2 a_{2} b_{2}\right)\right]^{1 / 2}
\end{aligned}
$$

where $\quad a_{1}=R-R \sin \theta_{D} / \cos \varphi_{0}, \quad b_{1}=R\left[\theta_{D}-\cos ^{-1}(1-(D-f) / R]\right.$, $c_{1}=R\left[\sin \varphi_{0}-(1-(D-f) / R]\right.$,

$b_{2}=R_{W}\left[\xi_{1}-\xi_{2}+\sin ^{-1}\left(f \cos \theta_{\mathrm{p}} / e_{2}\right)\right]$ $a_{2}=R\left(1-\sin \theta_{E} / \sin \gamma\right)$, $R\left\{\theta_{\mathrm{E}}+\pi / 2-\cos ^{-1}\left[\frac{R_{\mathrm{W}}^{2}-R^{2}-e_{2}^{2}}{2 e_{2} R}\right]-\theta_{\mathrm{p}}\right\}$.

\section{Three-dimensional cutting forces}

To derive three-dimensional cutting forces, the experimental formulae of Ueda and Nakayama (Ueda and Matuso, 1986; Nakayama and Arai, 1976) were employed in modeling cutting geometry parameters $\phi_{\mathrm{e}}, \tau_{\mathrm{s}}$ and $\beta$ as a function of $\mathrm{V}, f, \alpha_{\mathrm{e}}$ in machining of 1045 steel with a HSS cutting tool such that:

$\phi_{\mathrm{e}}=106.7(\mathrm{~V} f)^{1 / 2}+0.375 \alpha_{\mathrm{e}}+13.64$

$\tau_{\mathrm{s}}=1.586(\mathrm{~V} f)^{-1 / 4}+67.703$

$\beta=48.4(\mathrm{Vf})^{1 / 8}+28.586-\phi_{\mathrm{e}}+\alpha_{\mathrm{e}}$

(V: $\mathrm{mm} / \mathrm{min} ; f: \mathrm{mm} /$ tooth; $\phi_{\mathrm{e}}, \beta$ : degree $\left(^{\circ}\right) ; \tau_{\mathrm{s}}: \mathrm{kgf} / \mathrm{mm}^{2}$ )where $\phi_{\mathrm{e}}$ is the effective shear angle and $\alpha_{\mathrm{e}}$ is the effective rake angle. The angle $\alpha_{\mathrm{e}}$ can be expressed as

$\alpha_{\mathrm{e}}=\sin ^{-1}\left(\sin \alpha_{\mathrm{r}} \sin i \cos \eta_{\mathrm{c}}+\sin \eta_{\mathrm{c}} \sin i\right)$

Based on Eqs. (22)-(24), the shear energy per unit time $U_{s}$ can be written in terms of $A_{s}, \tau_{s}, V, \alpha_{e}$ and $\phi_{\mathrm{e}}$ :

$\mathrm{U}_{\mathrm{S}}=\tau_{\mathrm{s}} A_{\mathrm{s}} \mathrm{V}\left[\frac{\cos \alpha_{e}}{\cos \left(\phi_{\mathrm{e}}-\alpha_{\mathrm{e}}\right)}\right]$

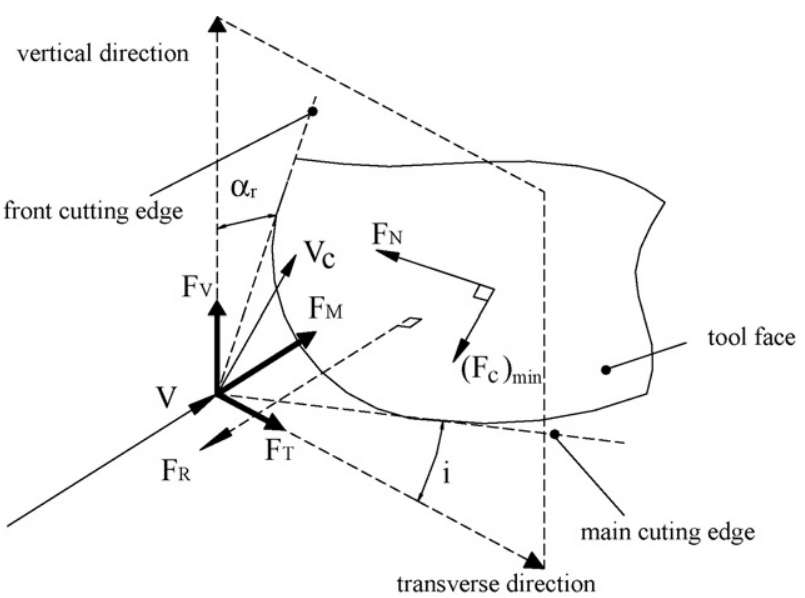

Fig. 6 - The directions of $F_{R}, F_{N},\left(F_{f}\right)_{\min }, F_{M}, F_{V}$ and $F_{T}$ in three-dimensional static tool axis system.

and the friction energy per unit time $U_{\mathrm{f}}$ can be expressed as a function of $Q_{p}, \tau_{\mathrm{s}}, V, \alpha_{\mathrm{e}}$ and $\phi_{\mathrm{e}}$ :

$U_{\mathrm{f}}=\tau_{\mathrm{s}} \mathrm{Q}_{\mathrm{p}} \mathrm{V}\left[\frac{\sin \phi_{e}}{\cos \left(\phi_{\mathrm{e}}-\alpha_{\mathrm{e}}\right)}\right]$

Since $U$ is the sum of $U_{\mathrm{s}}$ and $U_{\mathrm{f}}$, it is concluded that $U$ is a function of $A_{s}, Q_{p}, V, \alpha_{e}, \phi_{e}$ and $\tau_{s}$. By Usui's concepts, the $\eta_{c}$ is a value that result in minimum cutting energy per unit time, $U_{\text {min }}$. Substituting this $\eta_{\mathrm{c}}$ into Eq. (25), the rake angle $\alpha_{\mathrm{e}}$ at the minimum cutting energy per unit time is obtained. Because that effective frictional area $Q_{p}$ is a function of $\alpha_{e}$, a frictional force $\left(F_{c}\right)_{\min }$ in Eq. (10) can be calculated. Therefore, a main cutting force $F_{M}$ is obtained

$F_{\mathrm{M}}=\frac{U_{\min }}{V}$

The resultant force, $F_{R}$, which consists of frictional force $\left(F_{C}\right)_{\min }$, and normal force on the tool face, $F_{N}$, is equal to the main cutting force, $F_{M}$, that it,

$F_{\mathrm{M}}=F_{\mathrm{N}} \cos \alpha_{\mathrm{r}} \cos i+\left(F_{\mathrm{c}}\right)_{\min } \sin \alpha_{\mathrm{e}}$

Normal force on the tool face, $F_{N}$, is determined from equation (29), that is,

$F_{N}=\frac{F_{M}-\left(F_{C}\right)_{\min } \sin \alpha_{\mathrm{e}}}{\cos \alpha_{\mathrm{r}} \cos i}$

Then, the transverse component of cutting force, $F_{\mathrm{T}}$, and the vertical component of cutting force, $F_{\mathrm{V}}$, are determined respectively as

$F_{\mathrm{T}}=\left(F_{\mathrm{C}}\right)_{\min }\left(\sin \eta_{\mathrm{C}} \cos i-\sin \alpha_{\mathrm{r}} \sin i \cos \eta_{\mathrm{c}}\right)-F_{\mathrm{N}} \cos \alpha_{\mathrm{r}} \sin i$

$F_{\mathrm{V}}=\left(F_{\mathrm{C}}\right)_{\min } \cos \alpha_{\mathrm{r}} \cos \eta_{\mathrm{C}}-F_{\mathrm{N}} \sin \alpha_{\mathrm{r}}$

The directions of $F_{R}, F_{N},\left(F_{c}\right)_{\min }, F_{M}, F_{V}$ and $F_{T}$ in threedimensional static tool axis system are shown in Fig. 6.

Because of the revolution of ball-end cutter, the threedimensional cutting forces are influenced by axial depth of cut, 
direction of feed, instantaneous rational angle and the indentation effect of the tool tip. Taking these effects into account, the cutting forces are written as a matrix form:

$\mathbf{F}=\mathrm{T}_{\mathrm{HB}} \mathrm{T}(\Omega) \mathrm{T}_{\delta} \mathrm{T}_{\theta} \mathrm{T}_{\varepsilon} \mathrm{F}^{*}$

where $\mathrm{F}^{*}$ is the original three-dimensional cutting forces matrix in a static tool axis system obtained by Eqs. (28), (31) and (32), that is,

$F^{*}=\left[\begin{array}{c}F_{M} \\ F_{V} \\ F_{T}\end{array}\right]$

$\mathrm{T}_{\varepsilon}$ is a transformation matrix due to the influence of axial depth of cut, that is,

$\mathrm{T}_{\varepsilon}=\left[\begin{array}{ccc}0 & -\cos \varepsilon & \sin \varepsilon \\ 1 & 0 & 0 \\ 0 & \sin \varepsilon & \cos \varepsilon\end{array}\right]$

where $\varepsilon$ is expressed as

$\varepsilon=\pi / 4+\frac{1}{2} \sin ^{-1}[(D-R) / R]$

$\mathbf{T}_{\theta}$ is a transformation matrix owing to the variation of feeding direction during cutting process, that is,

$\mathbf{T}_{\theta}=\left[\begin{array}{ccc}\cos \theta & 0 & \sin \theta \\ 0 & 1 & 0 \\ -\sin \theta & 0 & \cos \theta\end{array}\right]$

where $\theta$ is the feed angle. When feeding in horizontal and vertical direction, $\theta$ is substituted by $0^{\circ}$ and $90^{\circ}$, respectively.

$\mathrm{T}_{\delta}$ is a transformation matrix caused by the effect of helix angle $\delta$, that is,

$\mathrm{T}_{\delta}=\left[\begin{array}{ccc}1 & 0 & 0 \\ 0 & \cos \delta & \sin \delta \\ 0 & -\sin \delta & \cos \delta\end{array}\right]$

A transformation matrix of tool rotational angle $\mathrm{T}_{\Omega}(\Omega)$ can be expressed as

$\mathrm{T}_{\Omega}(\Omega)=\left[\begin{array}{ccc}\cos \Omega & -\sin \Omega & 0 \\ \sin \Omega & \cos \Omega & 0 \\ 0 & 0 & 1\end{array}\right]$

where $\Omega$ is the tool rotational angle. The influence of $\Omega$ is different for horizontal cutting and vertical cutting. For the two-flute cutter the transformation matrix is:

(1) for horizontal cutting, $\mathrm{T}_{\Omega}(\Omega)=\mathrm{T}_{\Omega \mathrm{H} 2}(\Omega)$

$$
\mathrm{T}_{\Omega \mathrm{H} 2}(\Omega)=\mathrm{T}_{\Omega}(j)+\mathrm{T}_{\Omega}\left(\mathrm{j}^{*}\right)
$$

when $j=0-180^{\circ}+\lambda$ and $j^{*}=180-360^{\circ}, \mathrm{T}_{\Omega}(j)=[0]$, when $j>180^{\circ}+\lambda, \mathrm{T}_{\Omega}\left(j^{*}\right)=[0]$, when $j^{*}<180^{\circ}$, where $\lambda$ is given by the following equation:

$$
\lambda=2 \sin ^{-1}\left[\frac{D \tan (\delta-\psi)}{2 R}\right]
$$

(2) for vertical cutting, $\mathrm{T}_{\Omega}(\Omega)=\mathrm{T}_{\Omega \mathrm{V} 2}(\Omega)$

where $\mathrm{T}_{\boldsymbol{\Omega} \mathrm{V} 2}(\Omega)=\mathrm{T}_{\boldsymbol{\Omega}}(m)+\mathrm{T}_{\boldsymbol{\Omega}}\left(m+180^{\circ}\right)$

for $m=0 \sim 360^{\circ}$

The cutting force of four-flute cutter can be taken as the superposition of the cutting forces of two two-flute cutters with $90^{\circ}$ phase difference. Hence, for four-flute cutter the transformation matrix of rotational angle is:

(1) for horizontal cutting, $\mathrm{T}_{\Omega}(\Omega)=\mathrm{T}_{\Omega \mathrm{H} 4}(\Omega)$

where $\mathrm{T}_{\Omega \mathrm{H} 4}(\Omega)=\mathrm{T}_{\Omega \mathrm{H} 2}(k)+\mathrm{T}_{\Omega \mathrm{H} 2}\left(k+90^{\circ}\right)$

for $k=0 \sim 360^{\circ}$

(2) for vertical cutting, $\mathrm{T}_{\Omega}(\Omega)=\mathrm{T}_{\Omega \mathrm{V} 4}(\Omega)$

where $\mathrm{T}_{\Omega \mathrm{V} 4}(\Omega)=\mathrm{T}_{\Omega \mathrm{V} 2}(n)+\mathrm{T}_{\Omega \mathrm{V} 2}\left(n+90^{\circ}\right)$

for $n=0 \sim 360^{\circ}$

$\mathrm{T}_{\mathrm{HB}}$ is a transformation matrix to account for the effects of indentation force of tool tip in $Z$ direction, that is,

$T_{H B}=\left[\begin{array}{ccc}1 & 0 & 0 \\ 0 & 1 & 0 \\ 0 & 0 & H B \pi L_{\text {in }}^{2}\end{array}\right]$

where $L_{\text {in }}=R \sin (\psi)$ in the horizontal direction, $L_{\text {in }}=R \sin \left(\pi / 2-\varphi_{0}\right)$ in the vertical direction. $H B$ is the Brinell hardness of material.

$\mathbf{F}$ is a three-dimensional cutting matrix in Cartesian system, that is,

$F=\left[\begin{array}{c}F_{X} \\ F_{Y} \\ F_{Z}\end{array}\right]$

\section{Experiments and discussion}

To verify the proposed force model in horizontal and vertical direction cutting process, more than 100 cutting tests under various cutting conditions were conducted on a three-axis vertical CNC milling machine. The spindle speed was controlled at $1500 \mathrm{rpm}$. Two kinds of HSS cutting tools having $30^{\circ}$ helix angle and $40 \mathrm{~mm}$ length were used in the experiment. One was two flutes of $8 \mathrm{~mm}$ in diameter and the other was four flutes of $16 \mathrm{~mm}$ diameter. The workpiece material is a block of 1045 steel. A table type Kistler dynamometer was used to measure 

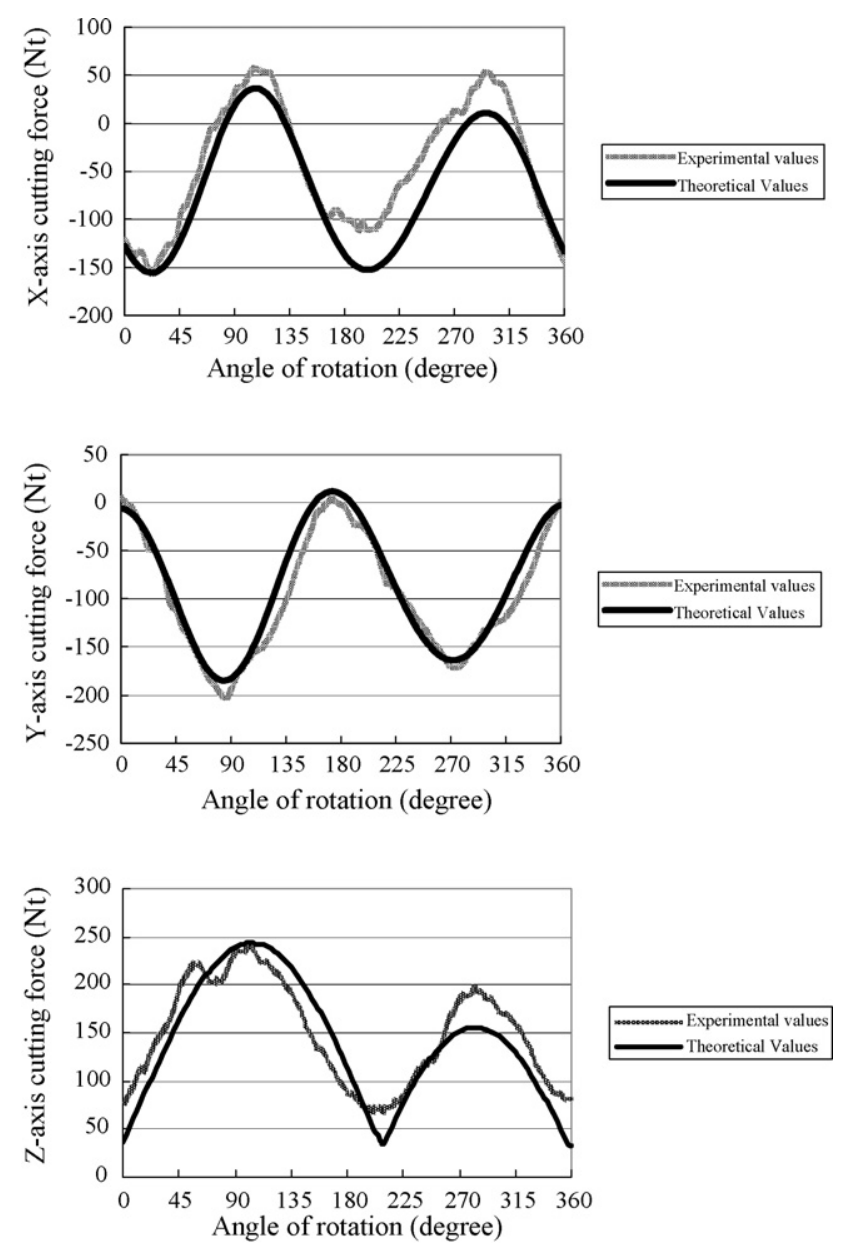

Fig. 7 - The experimental and theoretical cutting forces with two-flute and R4 ball-end cutter for horizontal feed rate $40 \mathrm{~mm} / \mathrm{min}$ and cutting depth $2 \mathrm{~mm}$.

the $\mathrm{X}, \mathrm{Y}$ and $\mathrm{Z}$ direction cutting forces. The experiments were conducted with no cutting fluid applied.

The measured and predicted cutting forces under various cutting conditions and tools are shown in Figs. 7-9. In Fig. 7 the tool used is a two-flute and R4 ball-end cutter, it is fed horizontally with a rate of $40 \mathrm{~mm} / \mathrm{min}$ and a cutting depth of $2 \mathrm{~mm}$. Fig. 8 shows the results along vertical direction cutting with a feed rate of $6 \mathrm{~mm} / \mathrm{min}$ and a cutting depth of $2 \mathrm{~mm}$. In Fig. 9, cutting is performed along horizontal direction by a four-flute and R8 ball-end cutter with a feed rate of $20 \mathrm{~mm} / \mathrm{min}$ and a cutting depth of $2 \mathrm{~mm}$. It can be seen from these figures that the trends and profiles of predicted forces agree with experimental ones both in horizontal and vertical cuts. This indicates that the presented cutting force model and the assumption of taking an average cutting velocity for the whole cutting edge in the model are acceptable. Even though there are some departures between the measurements and predictions, the force model is accurate enough for the purpose of explaining the cutting phenomena since the characteristics of the cutting forces still inhere in the model. The agreement between the predicted and experimental data in Fig. 9 also confirms the postulation that the cutting forces of four-flute cutter can be obtained by superposition of two two-flute cutting force
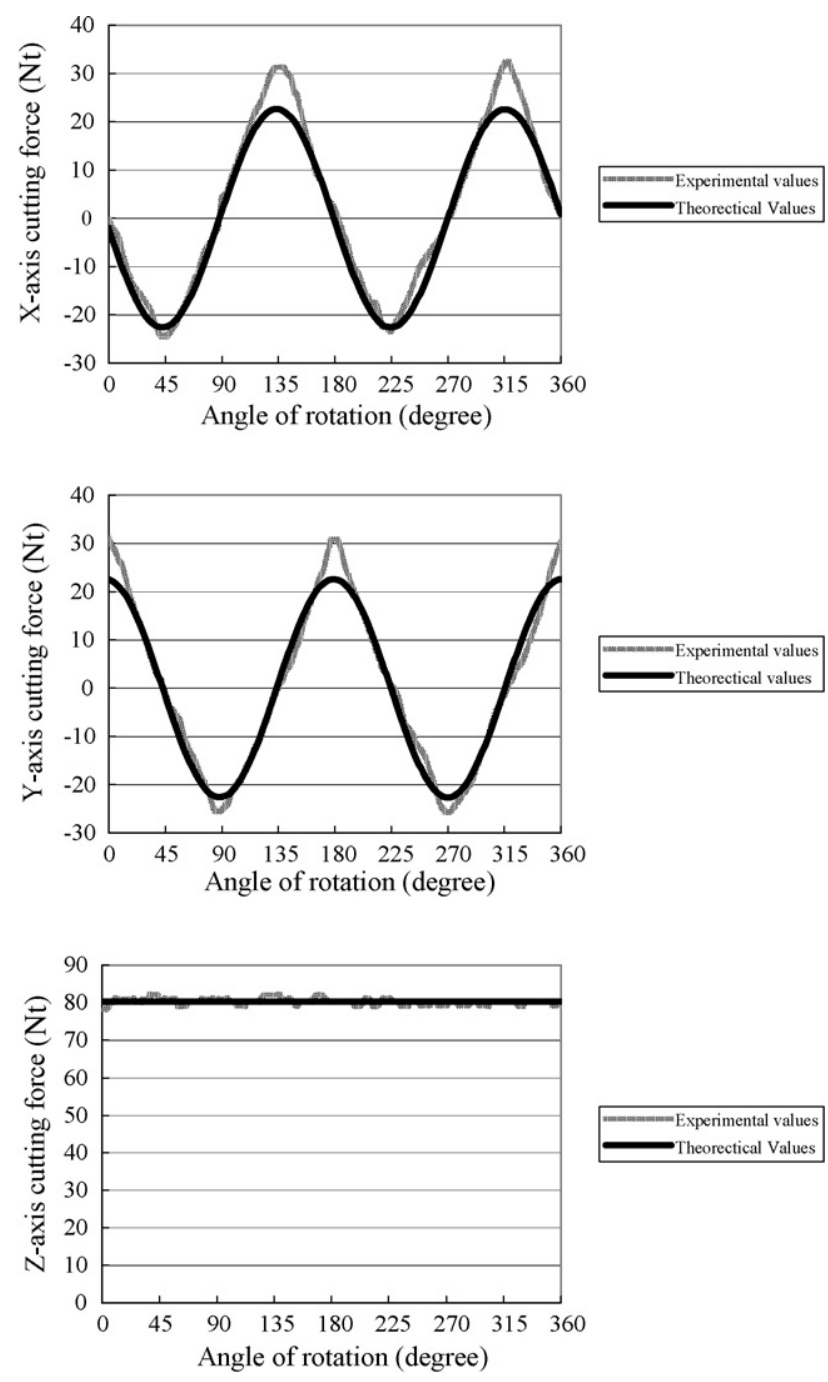

Fig. 8 - The experimental and theoretical cutting forces with two-flute and R4 ball-end cutter for vertical feed rate $6 \mathrm{~mm} / \mathrm{min}$ and cutting depth $2 \mathrm{~mm}$.

model having 90 degrees phase difference. Thus, the theoretical forces are taken to study the variations of three axes cutting forces.

The theoretical three-dimensional cutting forces for twoflute cutting along horizontal direction are shown in Fig. 10. The $\mathrm{X}, \mathrm{Y}$ and $\mathrm{Z}$ direction forces behave as a sinusoidal wave. Due to the decomposition of cutting force on the cutting edge induced by the revolution of the cutter described in tool rotation angle $\Omega$ of matrix $\mathrm{T}_{\Omega}(\Omega)$ of Eq. (39), the $X$ direction force has a phase lead over that of $Y$ direction's. Most of the $X$ and $Y$ direction forces are negative in horizontal feed. The small portion of positive force is as shown in the figure. This is due to the cutting action of a small cutting edge opposite to main cutting edge. As a result, the center-leading angle $\psi$ shown in Fig. 4 is formed in the opposite side of main cutting edge and this causes the positive force different from the negative force of main cutting edge(Moreover, it can be seen from Fig. 10 that the $Z$-axis force shifts up to a higher value. This is because that an increase of angle $\psi$ on tool tip will induce extra indentation 

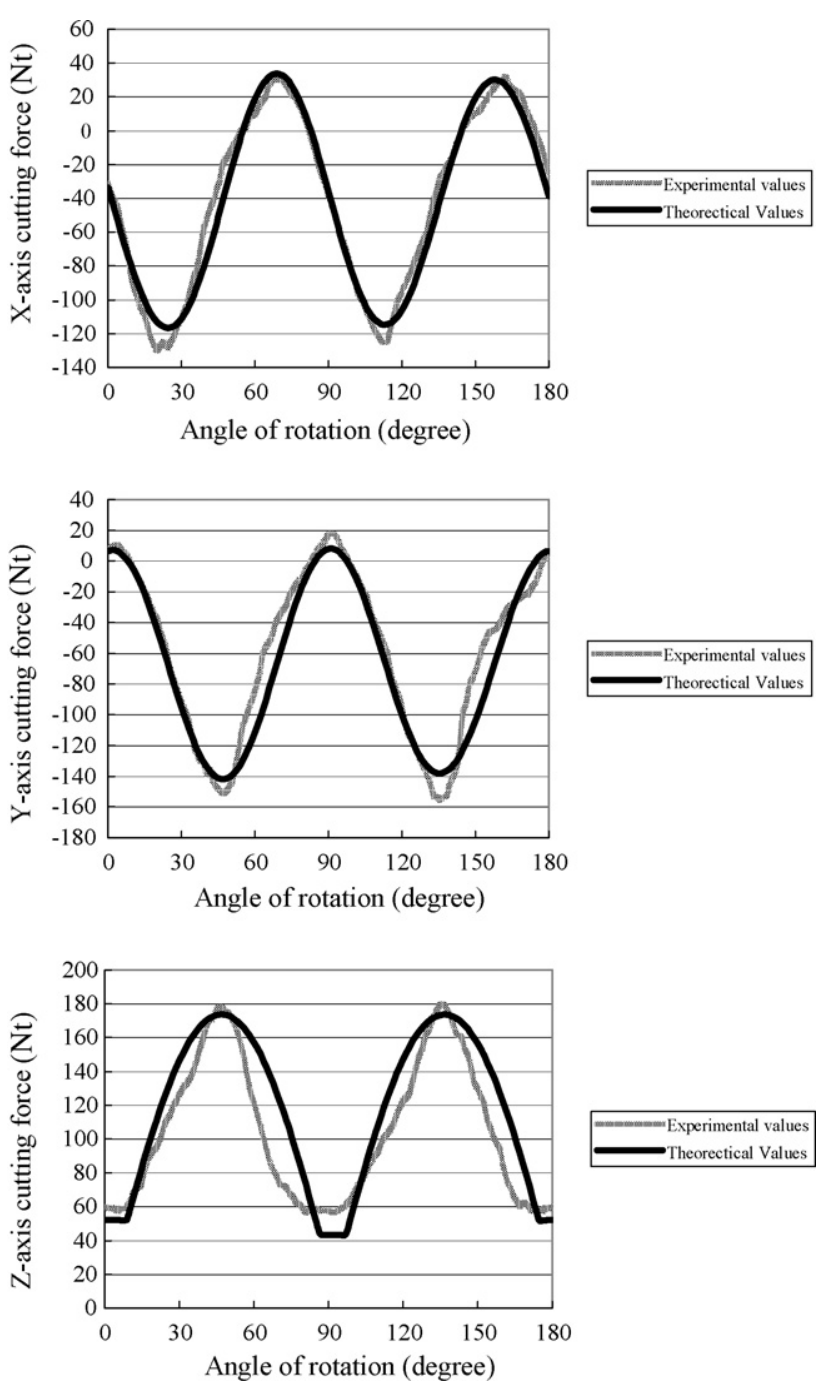

Fig. 9 - The experimental and theoretical cutting forces with four-flute and R8 ball-end cutter for horizontal feed rate $20 \mathrm{~mm} / \mathrm{min}$ and cutting depth $2 \mathrm{~mm}$.

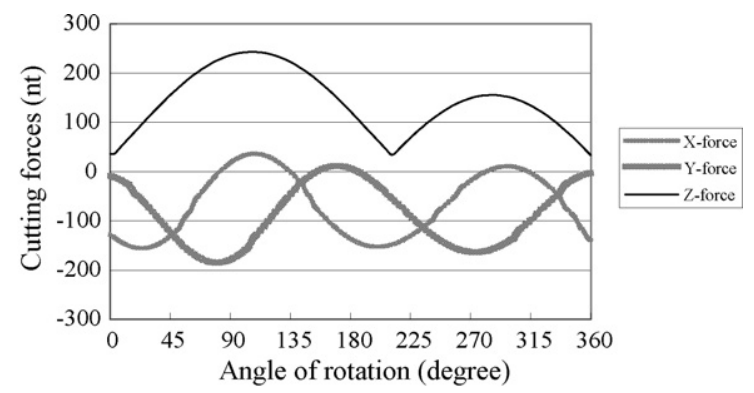

Fig. 10 - Theoretical three-axis cutting forces of Fig. 7.

force added to $Z$-axis force. It can be verified by transformation matrix of tool tip indentation effect $\mathrm{T}_{\mathrm{HB}}$ in equation (40).

Fig. 11 shows the theoretical cutting forces of a two-flute cutter in vertical direction cutting. The characteristics of the sinusoidal waveform of $\mathrm{X}$-axis and $\mathrm{Y}$-axis forces in vertical feed cutting are similar to those of the horizontal feed cutting

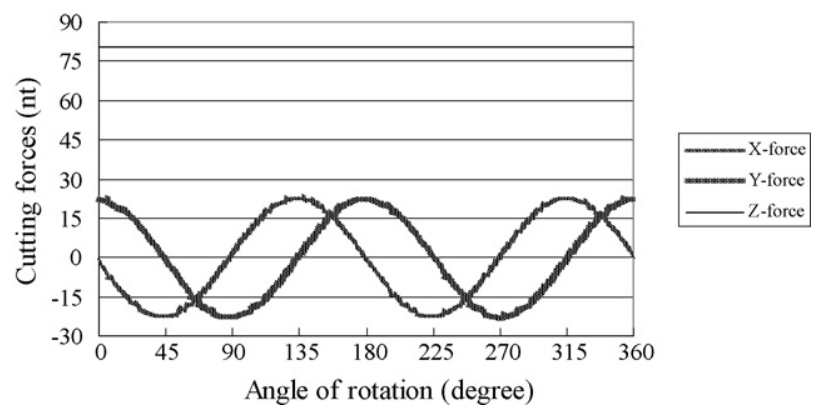

Fig. 11 - Theoretical three-axis cutting forces of Fig. 8.

described in Fig. 10. The Z-axis force is about a straight line, and it is completely different from the sinusoidal waveform of Z-axis force along horizontal feed cutting. In horizontal direction cutting, there is an intermittent cutting action of one cutting edge after another during half revolution, so that the cutting forces are varied in one revolution of the cutter. However, for vertical direction cutting, two cutting edges cut simultaneously during one revolution of the cutter. In this case, the superposition of two opposite and symmetric forces with respect to tool axis results in a roughly constant $Z$-axis force. It can be explained by the matrix of $\mathrm{T}_{\Omega \mathrm{V} 2}(\Omega)$ shown in Eq. (39.3). Furthermore, it can be seen that the value of the $Z$-axis force is far larger than zero. This is because of a larger squeeze effect of indentation force (i.e. a larger center-leading angle $\psi$ ) on tool tip resulting from a squeeze line of "ndj" as shown in Fig. 3.

Fig. 12 shows the theoretical forces of a four-flute cutter in horizontal feed cutting. The trends of $X$ - and $Y$-axis forces behave as those of two-flute cutters. But for $Z$-axis force, there is constant force between two consecutive waves. This is attributed to the fact that two cutting edges perform cutting action simultaneously during every $90^{\circ}$ of cutting range, so that there is a force wave during every $90^{\circ}$-rotation angle. The superposition of cutting forces can be presented as a matrix $\mathrm{T}_{\Omega \mathrm{H} 4}(\Omega)$ in Eq. (39.4). However, the former force wave will interfere with the next force wave with a cutting engage range of $\psi$. When the cutting forces are superposed at these points, the $\mathrm{Z}$-axis force reaches the lowest value and it is about constant as shown in Fig. 12.

Based on the above geometric analysis, the model presented can be applied both in two and four-flute milling process in the horizontal feeding cutting. The cutting forces

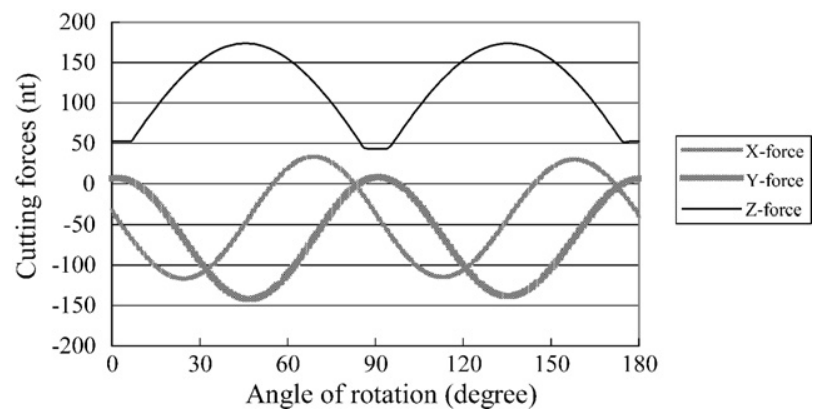

Fig. 12 - Theoretical three-axis cutting forces of Fig. 9. 
of the four-flute ball-end cutter feeding in the vertical direction can also be superposed by two two-flute forces, and they will be nearly the same as two-flute ones shown in Fig. 11.

\section{Conclusion}

A new geometric force model of ball-end cutter, which can predict three-dimensional cutting forces, is presented in this paper. An assumed complete cutting edge, that is different from the previous researches that the cutting edge was divided into infinitesimal straight unit, is taken into account. The key parameters on the cutting forces, such as tool rational angle and center-leading angle, are introduced to force transformation matrix by means of geometric analysis. The different engaged region is the reason that causes difference in amplitude and shape of the cutting force. The squeeze effect on tool tip and a higher center-leading angle result in a higher value of $Z$-direction force. The symmetrical sinusoidal waveform of $X$, $Y$ direction forces with respect to zero-force axis and an almost constant value of $Z$-direction force in vertical direction cutting can be described with geometry analysis and explained by the proposed model. The cutting forces of four-flute cutter can be superposed by the two-flute ones having $90^{\circ}$ phase difference. The presented model can be treated as a basic model. In the next paper of this work, a geometric model along nonhorizontal cutting for the analysis of ball-end milling will be presented. Furthermore, any type of cutting tool with or without nose radius at the tool tip, single- or multi-flute of cutting edge, and flat- or ball-end milling tool, can be easily analyzed and synthesized by the proposed model, and this research is under way.

\section{Appendix A}

As described in the test of Eq. (16),

$A_{s, H}=A_{s 1, H}+A_{s 2, H}=\int_{\varphi_{2}}^{\varphi_{1}} f_{1}(\varphi) d s+\int_{\varphi_{0}}^{\varphi_{2}} f_{2}(\varphi) d s$

Functions of $f_{1}(\varphi)$ and $f_{2}(\varphi)$ are computed as follows:

$f_{1}(\varphi)=a_{3}+b_{3}-\left(R^{2}-c_{3}^{2}\right)^{1 / 2}$

where

$a_{3}=(f+R \cos \varphi) \cos \eta_{\mathrm{cP}}$

$b_{3}=R \sin \varphi \sin \eta_{\mathrm{CP}}$

$c_{3}=\left|(f+R \cos \varphi) \cos \eta_{\mathrm{CP}}-R \sin \varphi \cos \eta_{\mathrm{CP}}\right|$

$f_{2}(\varphi)=\frac{f_{1}\left(\varphi_{2}\right)-R\left(\sin \varphi_{2}-\sin \varphi\right)}{\sin \eta_{\mathrm{CP}}}$

The calculus variables $\varphi_{0}$ and $\varphi_{1}$ can be derived by the following equations:

$\varphi_{0}=\sin ^{-1}\left[\frac{(R-D)}{R}\right]$ $\varphi_{1}=\frac{\pi}{2}+\sin ^{-1}\left(\frac{f}{2 R}\right)$

while $\varphi_{2}$ is determined so as to satisfy the following equation:

$R-D=R \sin \varphi-f_{1}\left(\varphi_{2}\right) \sin \eta_{C P}$

In addition, Eq. (20) in the test,

$A_{\mathrm{s}, \mathrm{V}}=A_{\mathrm{s} 1, \mathrm{~V}}+A_{\mathrm{s} 2, \mathrm{~V}}+A_{\mathrm{s} 3, \mathrm{~V}}$

$=\int_{\varphi_{2}}^{\varphi_{1}} g_{1}(\varphi) \mathrm{d} s+\int_{\varphi_{3}}^{\varphi_{2}} g_{2}(\varphi)+\int_{\varphi_{0}}^{\varphi_{3}} g_{3}(\varphi) \mathrm{d} s$

Functions of $g_{1}(\varphi), g_{2}(\varphi)$ and $g_{3}(\varphi)$ are given by equations (20a), (20b) and (20c) described below:

$g_{1}(\varphi)=a_{4}+b_{4}-c_{4}$

where

$a_{4}=(f+R \sin \varphi) \sin \eta_{\mathrm{CP}}$

$b_{4}=R \cos \varphi \cos \eta_{\mathrm{CP}}$

$c_{4}=\left(\frac{R_{\mathrm{W}}-e_{2} \sin \theta_{\mathrm{A}}}{\sin \theta_{\mathrm{B}}}\right) \cos \theta_{\mathrm{C}}$

In the above equations, the variables $\theta_{\mathrm{A}}, \theta_{\mathrm{B}}$ and $\theta_{\mathrm{C}}$ can be expressed by the following equations:

$\theta_{\mathrm{A}}=\frac{\pi}{2}-\eta_{\mathrm{CP}}+\sin ^{-1}\left(\frac{f \sin \theta_{\mathrm{p}}}{e_{2}}\right)+\theta_{\mathrm{p}}$

$\theta_{\mathrm{B}}=\pi / 2-\frac{\varphi-\varphi_{2}}{\left(\varphi_{1}-\varphi_{2}\right) \xi_{1}} \xi_{2}+\eta_{\mathrm{CP}} \sin ^{-1}\left(\frac{f \sin \theta_{\mathrm{p}}}{e_{2}}\right) \theta_{\mathrm{p}}$

$\theta_{\mathrm{C}}=\frac{\left(\varphi-\varphi_{2}\right)}{\left(\varphi_{1}-\varphi_{2}\right) \xi_{1}}+\eta_{\mathrm{CP}} \xi_{2}+\sin ^{-1}\left(\frac{f \sin \theta_{\mathrm{p}}}{e_{2}}\right)+\theta_{\mathrm{p}}$

where

$\xi_{1}=\cos ^{-1}\left[\frac{e_{1}^{2}+R_{\mathrm{W}}^{2}-R^{2}}{2 e_{1} R_{\mathrm{W}}}\right]$

$\xi_{2}=\cos ^{-1}\left[\frac{e_{2}^{2}+R_{\mathrm{W}}^{2}-R^{2}}{2 e_{2} R_{\mathrm{W}}}\right]$

$g_{2}(\varphi)=a_{5}+b_{5}-\left(R^{2}-c_{5}^{2}\right)^{1 / 2}$

where

$a_{5}=(f+R \sin \varphi) \sin \eta_{\mathrm{CP}}$

$b_{5}=R \cos \varphi \cos \eta_{\mathrm{cP}}$

$C_{5}=\left|(f+R \sin \varphi) \cos \eta_{\mathrm{CP}}-R \cos \varphi \sin \eta_{\mathrm{CP}}\right|$

$g_{3}(\varphi)=\frac{g_{2}\left(\varphi_{3}\right)-R\left(\sin \varphi_{3}-\sin \varphi\right)}{\sin \eta_{\mathrm{CP}}}$ 
The variables, $\varphi_{0}$ and $\varphi_{1}$ can be derived by the following equations:

$\varphi_{0}=\sin ^{-1}\left[\frac{(R-D)}{R}\right]$

$\varphi_{1}=\theta_{\mathrm{p}}+\cos ^{-1}\left[\frac{\mathrm{R}_{\mathrm{W}}^{2}-\mathrm{R}^{2}-e_{1}^{2}}{2 e_{1} R}\right]$

while $\varphi_{2}$ and $\varphi_{3}$ are determined so as to satisfy the following equations:

$e_{1}+R \cos \left(\varphi_{2}-\theta_{\mathrm{p}}\right)=\frac{R \sin \left(\varphi_{2}-\theta_{\mathrm{p}}\right)}{\tan \left(\theta_{\mathrm{CP}}-\theta_{\mathrm{p}}\right)}-\frac{R_{\mathrm{W}} \sin \left(\xi_{2}+\theta_{\mathrm{D}}+\theta_{\mathrm{p}}-\eta_{\mathrm{CP}}\right)}{\sin \left(\eta_{\mathrm{CP}}-\theta_{\mathrm{p}}\right)}$

$R-D=R \sin \varphi-g_{2}(\varphi) \sin \eta_{c P}$

\section{REFERENCES}

Engin, S., Altintas, Y., 2001. Mechanics and dynamics of general milling cutters. Part I. Helical end mills. Int. J. Machine Tools Manuf. 41, 2195-2212.
Fontaine, M., Devillez, A., Moufki, A., Dudzinski, D., 2006. Predictive force model for ball-end milling and experimental validation with a wavelike form machining test. Int. J. Machine Tools Manuf. 46, 367-380.

Kivanc, E.B., Budak, E., 2004. Structural modeling of end mills for form error and stability analysis. Int. J. Machine Tools Manuf. 44, 1151-1161.

Lee, P., Altintas, Y., 1996. Prediction of ball-end milling forces from orthogonal cutting data. Int. J. Machine Tools Manuf. 36, 1059-1072.

Nakayama, K., Arai, M., 1976. On the storage of data on metal cutting forces. CIRP Annals 25, 13-18.

Shaw, M.C., 1984. Metal Cutting Principles. Clarendon Press, Oxford.

Ueda, N., Matuso, T., 1986. An investigation of some shear angle theories. CIRP Annals 35, 27-30.

Usui, E., Hirota, A., 1978. Analytical prediction of three dimensional cutting process. Part 2. Chip formation and cutting force with conventional single-point tool. Trans. ASME, J. Eng. Ind. 100, 229-235.

Usui, E., Hirota, A., Masuko, M., 1978. Analytical prediction of three dimensional cutting process. Part 1: basic cutiing model and energy approach. Trans. ASME, J. Eng. Ind. 100, 222-228.

Yang, M., Park, H., 1991. The prediction of cutting force in ball-end milling. Int. J. Machine Tools Manuf. 31 (1991), 45-54. 